\title{
SENSOR CALIBRATION USING NONPARAMETRIC STATISTICAL CHARACTERIZATION OF ERROR MODELS
}

\author{
J. Feng ${ }^{+}$, G. Qu ${ }^{*}$, M. Potkonjak ${ }^{+}$ \\ ${ }^{+}$Computer Science Dept. University of California, Los Angeles \\ 3532G Boelter Hall \\ Los Angeles, California 90034, USA \\ *Electrical and Computer Engineering Dept. University of Maryland \\ 1417 A.V. Williams \\ College Park, MD 20742, USA
}

\begin{abstract}
Calibration is the process of identifying and correcting for the systematic bias component of the error in sensor measurements. Traditionally, calibration has usually been conducted by considering a set of measurements in a single time frame and restricted to linear systems with the assumption of equal-quality sensors and single modality.

The basis for the new calibration procedure is to construct a statistical error model that captures the characteristics of the measurement errors. Such an error model can be constructed either off-line or on-line. It is derived using the nonparametric kernel density estimation techniques. We propose four alternatives to make the transition from the constructed error model to the calibration model, which is represented by piecewise polynomials. In addition, statistical validation and evaluation methods such as resubstitution, is used in order to establish the interval of confidence for both the error model and the calibration model. Traces of the distance ranging measurements recorded by in-field deployed sensors are used as our demonstrative example.
\end{abstract}

\section{INTRODUCTION}

One can distinguish the two components of error: systematic bias and random noise. Systematic bias is the time-invariant amplitude offset of the correct value and can be express by some deterministic function of the correct value. Random noise is time dependent and can be contributed by many factors such as environmental conditions and hardware noise. It is beneficial to separate the two components and address each individually, since each behaves differently. The random noise can be studied and modeled over time once the systematic bias is properly addressed.

In wireless sensor networks, calibration has been mainly addressed as a step of sensor fusion. Generally, it is common for the Gaussian distribution model to be assumed and the least linear-squares norm to be used in order to minimize discrepancy. However, a number of recent experiments indicate that errors in sensor systems do not follow the Gaussian distribution and often have complex behavior that cannot be captured using the standard distributions and parametric statistical methods. Our goal is to first study the error behavior using nonparametric statistical techniques where no assumption on error distribution is required. As we demonstrate with the distance (acoustic signal strength) measurements, the error behavior can only be accurately captured by nonparametric statistical techniques. The error model is constructed using the kernel density estimation technique. The approach is generic in that the error model can depend on an arbitrary number of measured parameters. Such an error model not only provides one single most probable correct value for a given measurement, it provides the complete probability distribution of all possible correct values for that single given measurement. Once the error model is available, we demonstrate that the calibration model used to map the recorded values to the correct values in order to compensate for sensor bias can be defined in several different ways. Furthermore, we are also able to derive the interval of confidence for any particular measurement value and for all values overall.

\section{RELATED WORK}

In this Section, we survey the related work along the following lines of statistical modeling and sensor data calibration. Least linear-squares fitting is one of the most popular parametric techniques for fitting a set of data to a particular compressed form. The technique is optimal under the assumption that the error distribution follows the Gaussian distribution. The standard technique for conducting least squares fitting is to use the Singular Value Decomposition (SVD) approach. In addition, there are a various available techniques that can be applied to determine whether the least squares model is appropriate of the given set of data. [3][1][8] provide the detailed description of these methods. In addition to parametric techniques, recently nonparametric techniques that do not depend on any underlying assumptions have been gaining popularity. The standard references include [4][5][6][11].

Calibration in sensor networks has a unique set of requirements and constraints due to the specifics of how sensor networks are deployed and operated. In particular, needs for low power operation and low bandwidth communication, harsh environments that accelerate degradation of sensors, and the cost of sensitivity pose unique challenges. Some of the existing state-of-the-art calibration techniques for sensor networks include [2][9][10][14][16][17]. Until now, calibration was addressed in sensor networks mainly in relationship to location discovery. The first two efforts that have been reported in this domain are Madusa system from UCLA and SpotON system from University of Washington. The emphasis in both efforts is on building models 
of the signal strength and the distance for a specific set of radio transceivers and receivers in off-line calibration. Both [9][14] reported comprehensive sets of experiments. Neither reports statistical evaluation of the developed models in terms of interval of confidence.

Another calibration project that targets location discovery is [17]. The authors use least linear-squares to fit experimental data to the manually selected linear model. The method has three phases. In the first step, they parameterize each individual radio and develop a model of the overall system using these parameters. In the second phase, they collect data from the system, and in the last phase they select the parameters for individual device in such a way that the behavior in the entire system has the lowest discrepancy. Several types of calibration techniques are discussed including iterative, mean, joint and autocalibration.

Bychkovskiy et al. [2] attempted to eliminate or reduce systematic error by first considering pairs of close sensors. During the next phase, they consider a large number of pairs of sensors and try to find the most consistent way to simultaneously satisfy all pairwise relationships. The basis for their work is an observation that two sensors that are spatially close often have temporarily correlated recordings.

More recently, Ihler et al. [10] proposed a localized selfcalibration approach that formulates the problem within a graphical model in the framework of location estimation. Then nonparametric belief propagation can applied to obtain an approximate solution. In addition, with the assumption that the noise in distance measurements follows the Gaussian distribution, self-calibration may be formulated as a nonlinear least-squares optimization problem.

\section{STATITICAL ERROR MODELING}

In this Section, we present the techniques to construct the nonparametric statistical model of the measurement errors represented in terms of the probability density functions. These techniques leverage the concept of kernel density estimation and the maximal likelihood principle. We first state the assumptions the techniques are based upon. Then we introduce the generic methodology for constructing the error model. After that, we provide evaluation metrics and validation techniques. We demonstrate the model construction process on a set of acoustic signal-based distance measurements (i.e. ranging measurements) generated by a set of deployed sensors [12][13]. It is important to note that these techniques are not limited to distance measurements, but also can be applied to a diverse range of data.

When the error model is constructed off-line, we assume the availability of golden standard, which can be either calculated by obeying physical laws (e.g. the distance formula in the case of distance measurements) or obtained by introducing additional calibrated and accurate equipment/sensors to serve as the reference values for the uncalibrated sensors. When the error model is constructed on-line and in field, no available standard is assumed. In this case, the system is first solved by using other optimization targets without explicitly specifying any error model (e.g. $\mathrm{L}_{1}$ norm, $\mathrm{L}_{2}$ norm), then the initial solutions serve as the correct values along with the measurements to construct the error model. After that, the system is solved again by adopting the online constructed error model. This process is repeated, and the error model is modified and tuned iteratively so that it is a better representation of the actual error distribution.

\subsection{Model Construction and Evaluation}

The distance ranging measurements that we use as the demonstrative example in this section was recorded by sensors deployed in a minefield test facility over the course of several days [12][13]. For the sake of simplicity, we explain the error construction off-line and the standard in this case is the calculated Euclidian distances for each measured distance given the coordinates of the sensors.

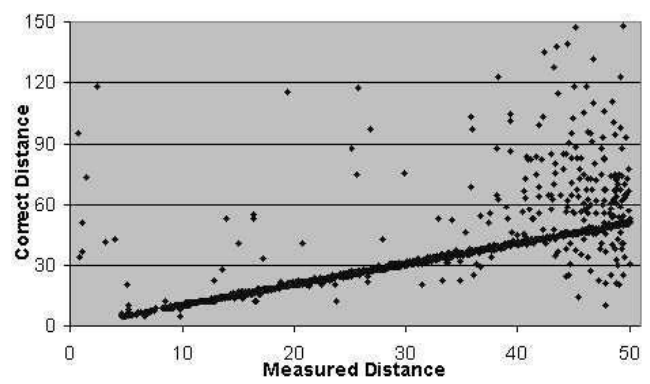

Figure 1. The measured vs. correct distances.

The first step towards building the error model is to examine the suitability of the measurements for modeling. The essence of suitability is to inspect whether there exists consistency among the measurements and to what extent the consistency is quantified. We first plot the pairs of measured and the corresponding calculated distance (i.e. the correct distances). Figure 1 shows 2,000 such pairs. In the case of distance measurements, we define consistency as longer measured distances imply longer corresponding correct distances. For this set of 2,000 data, the consistency is $92.93 \%$. Two observations show the consistency can be drawn from this figure: i) The majority of the measurements have low discrepancy when compared to the correct distance. ii) As the measured values increase, particularly when the measured distance $>40$, a substantially higher percentage of incorrect measurements is observed. Clearly, this set of distance measurements appears to be adequate for modeling since it has a high consistency and there are patterns and characteristics that can be generalized.

The essence of constructing measurement error model is to examine the frequency of different correct values given a single measured value. However, one difficulty towards constructing an accurate error model is the lack of sufficient number of measurement data. To address this limitation, the kernel density estimation technique [15] is used, where the probability of a measurement is estimated by considering other similar

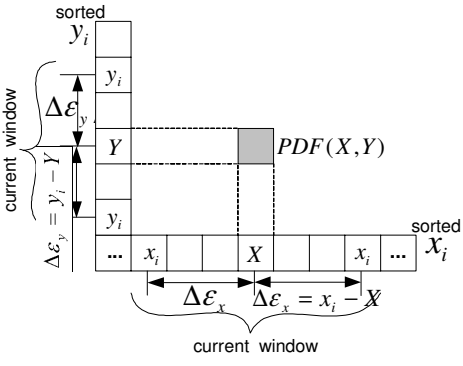

Figure 2. The similarity window.

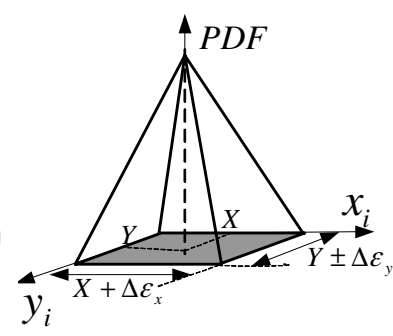

Figure 3. The kernel density estimation function. 


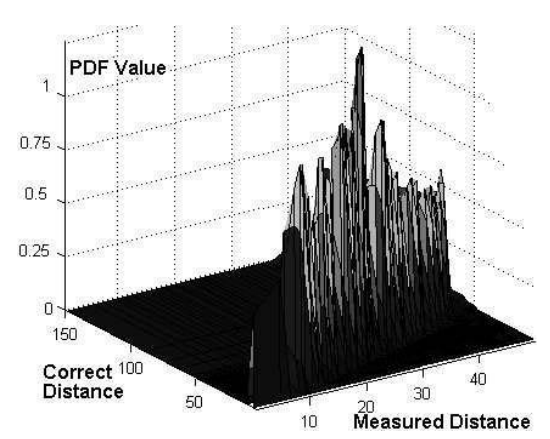

Figure 4(a). PDF surface.

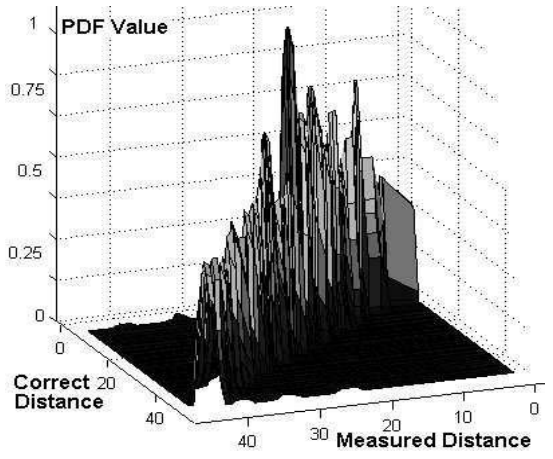

Figure 4(b). Close up view.

Measured distance $\in[0,40]$.

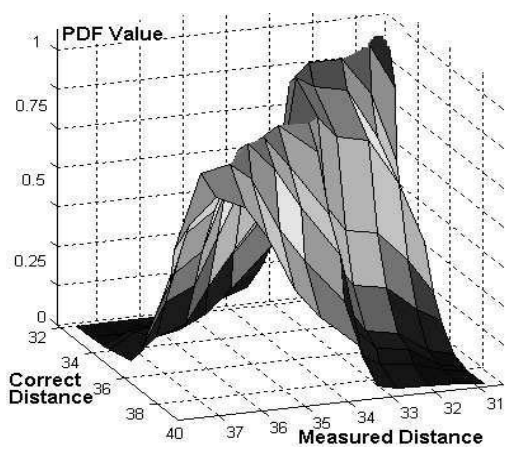

Figure 4(c). Close up view.

Measured distance $\in[30,40]$.

measurements. More specifically, we use 3-dimensional probability density functions (PDF) to represent the likelihood of errors. Consider a set of $n$ pairs of measured and correct distances $\left\{\left(x_{i}, y_{i}\right), i=1, \ldots, n\right\}$. For each measured distance $X$, we define a window of size $X \pm\left(\Delta \varepsilon_{x}\right)$, and consider all the measurements within this window to construct a 2-dimensional probability distribution curve of the correct distance for $X$. Furthermore, this 2-d curve is constructed by applying the same technique again on the correct distances. For each correct distance $Y$, we define another window of size $Y \pm\left(\Delta \varepsilon_{y}\right)$, all values fall within both of these two windows (i.e. $\left.\left\{\left(x_{i}, y_{i}\right) \mid x_{i} \in X \pm\left(\Delta \varepsilon_{x}\right), y_{i} \in Y \pm\left(\Delta \varepsilon_{y}\right)\right\}\right)$ are used to estimate the probability of the correct distance $Y$ given the measured distance $X$. The concept of similarity window is pictorially presented in Figure 2. Note that even values within the similarity windows, $\left\{\left(x_{i}, y_{i}\right) \mid x_{i} \in X \pm\left(\Delta \varepsilon_{x}\right), y_{i} \in Y \pm\left(\Delta \varepsilon_{y}\right)\right\}$, have different degrees of similarity with respect to $X$ and $Y$. Thus different weights should be assigned to $\left(x_{i}, y_{i}\right)$ according their similarity to $X$ and $Y$ towards estimating the probability of $(X, Y)$. Figure 3 gives an example of the possible weight function. The pyramid-shaped weight function has volume 1 , with the center of the pyramid being $(X, Y)$, and the sides being the window sizes $X \pm\left(\Delta \varepsilon_{x}\right)$ and $Y \pm\left(\Delta \varepsilon_{y}\right)$. Note that the further $\left(x_{i}, y_{i}\right)$ is from $(X, Y)$, the smaller weight is assigned to $\left(x_{i}\right.$, $\left.y_{i}\right)$. For all the values outside of either window, a weight zero is given which implies no contribution is made towards estimating the probability of $(X, Y)$. Figure 4(a) shows the 3-d PDF surface constructed upon the same set of 2,000 distance measurements using the pyramid weight function of window size 2.0. The $\mathrm{x}$-axis shows the measured distances; the y-axis shows the correct distances; and the z-axis indicates the likelihood of a particular correct distance for a particular measured distance. Figure 4(b) gives a closer view when the measured distance $\in[0,40]$; Figure 4 (c) gives a further closer view when the measured distance $\in[30$, 40].

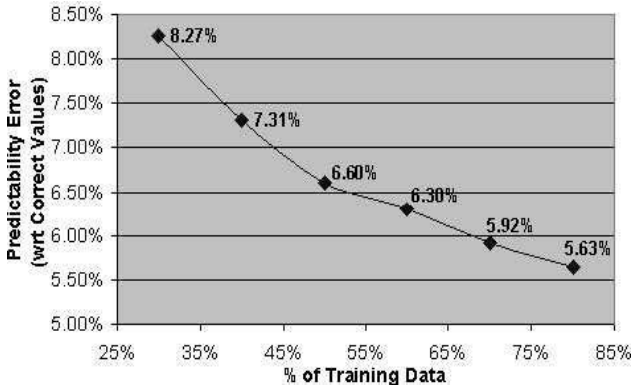

Figure 5. Predictability error given various $\%$ of the training data.

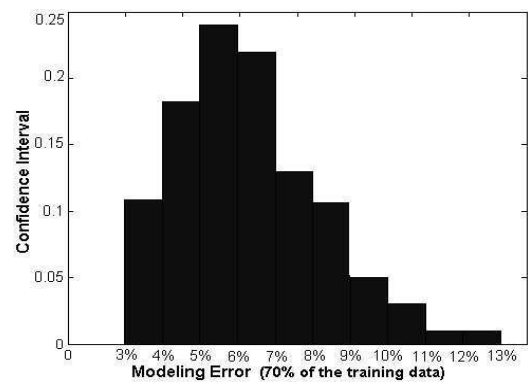

Figure 6. Consistency in terms of the interval of confidence.
The ultimate metric for evaluating the error model is the ability to accurately estimate the correct values that are not in the training data used to construct the error model. Therefore, it is crucial to study the relationship between the percentage of the training data and the modeling accuracy. Figure 5 shows the prediction error defined as the difference between the value proposed by the error model and the actual correct value, given different percentage of the training data varied from $30 \%$ to $80 \%$. As the plot indicates, even with only $30 \%$ of the training data, the error model still achieves only an error of $8.27 \%$ when compared to the correct values.

Another metric - consistency, provides a measure of how often the error model is within a certain bound of accuracy. It is done using the resubstitution method [5], where $70 \%$ of the original data is randomly selected to construct the error model, and the remaining $30 \%$ of the data is used to evaluate the model. This process is repeated 200 times to construct the interval of confidence. For example, Figure 6 shows the interval of confidence represented in terms of histograms of the prediction error when $70 \%$ of the training data is given. We can conclude from the figure that with $80 \%$ of confidence, the error model yields a prediction error of $5.5 \% \pm 1.5 \%$.

\section{CALIBRATION}

Another way to interpret the 3-d PDF surface is that it is a collection of 2-d PDF curves for each measured distance. For example, Figure 7 shows the 2-d PDF curve for the measured distance 30.26. This measured distance can be calibrated by selecting a correct distance based on this 2-d PDF plot. Given a measured distance $X$, we consider the following four alternatives:

1. Average: find the smallest (Min) and the largest (Max) correct distances that have PDF values greater than zero or a threshold; calculate the average of the two values.

2. Peak: select the real distance that has the highest PDF value.

3. 50\%: normalize the area under the 2-d PDF curve to 1 ; select the correct distance that partitions the area into two equal portions.

4. Weighted_Error: for each real distance $Y$, calculate the summation of weighted 
error defined as $\sum_{i=1}^{n}\left[\left|y_{i}-Y\right| \cdot \operatorname{PDF}\left(X, y_{i}\right)\right]$, where $y_{i}, i=1, \ldots, n$ are the correct distances; and $\operatorname{PDF}\left(X, y_{i}\right)$ is the $\operatorname{PDF}$ value of a specific real distance $y_{i}$ given the measured distance $X$.

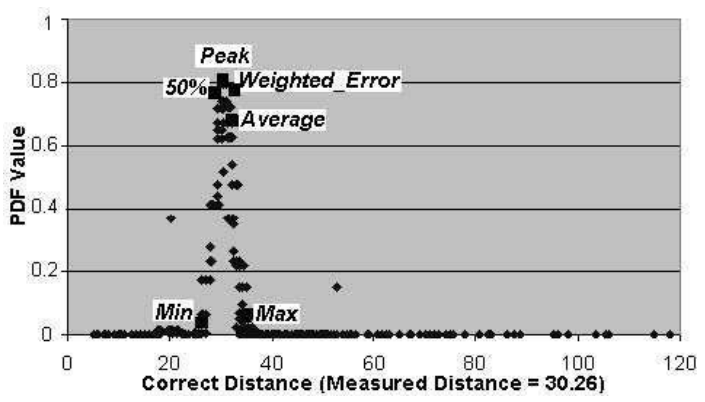

Figure 7. Probability density distribution of the correct distances of measured distance 30.26.

We first select the correct values (i.e. the calibrated values) for all measured distances based on one of the above four alternatives, then piece-wise polynomials are used to obtain an approximation of the calibration model using the least linear-squares. If the model is constructed on-line, a Dijkstra's shorted path-based algorithm [7] can be used to optimally determine the breaking point and the degree of each piece-wise polynomial function (Figure 8). Figure 9 shows the interval of confidence of the calibration error, which is defined as the difference between the correct values and the polynomial function estimate of the calibrated values. We can conclude from the figure that with $86 \%$ of the confidence, the calibration model, i.e. the two piece-wise polynomials, yields an error of $5.5 \% \pm 0.5 \%$.

\section{SUMMARY}

We have developed a calibration approach that employs the maximal likelihood principle for summarizing and representing the error model. The error model is derived using the nonparametric kernel density estimation techniques. Resubstitution validation method is applied in order to establish the interval of confidence for all results. We demonstrate the generic error model construction and evaluation techniques on a set of distance ranging measurements recorded by deployed sensors and the four calibration candidates. The calibration model is represented using piece-wise polynomial functions. Experimental results show not only that the statistical error model can accurately capture the behavior of the measurement errors with less than $50 \%$ of the training data; but also the calibration

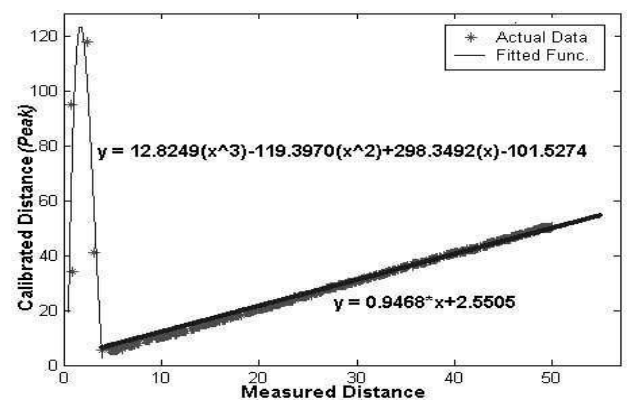

Figure 8. Calibration model presented as two piece-wise polynomials. model derived from the error model yields an average of $5.5 \%$ error with high confidence.

\section{REFERENCES}

[1] Belsley D.A., Kuh E., and Welsch R.E. Regression Diagnostics. New York, Wiley, New York, 1980.

[2] Bychkovskiy V., Megerian S., Estrin D., and Potkonjak M. "Colibration: A Collaborative Approach to In-Place Sensor Calibration". IPSN'03, Polo Alto, April 2003, pages 301316.

[3] Chatterjee S. and Price B. Regression Analysis by Example.Wiley, New York, 1977.

[4] Davison A.C. and Hinkley D.V. Bootstrap Methods and their Application. Cambridge University Press, Cambridge, UK, 1997.

[5] Efron B., The Jackknife, the Bootstrap, and Other Resampling Plans, Society for Industrial \& Applied Mathematics, 1982.

[6] Efron B. and Tibshirani R. An Introduction to the Bootstrap, Chapman-Hall, 1993.

[7] Feng J. and Potkonjak M, "Kernel Density Estimation-based Sensor Calibration", unpublished manuscript, 2004.

[8] Good P.I. Resampling Methods, Springer-Verlag, New York, NY, 1999.

[9] Hightower J., Vakili C., and Borriello G. "Design and Calibration of the SpotON Ad-Hoc Location Sensing System", UW CSE, University of Washington, August 2001.

[10] Ihler A.T. and Fisher J.W., "Nonparametric Belief Propagation for Self-Calibration in Sensor Networks", IPSN'04, Berkeley, April 2004, pages 225-233.

[11] Kahaner D., Moler C., and Nash S. Numerical Methods and Software. Prentice Hall, Englewood Cli.s, New Jersey, 1989.

[12] Merrill W., Girod L., Elson J., Sohrabi K., Newberg F., and Kaiser W., "Autonomous Position Location in Distributed, Embedded, Wireless Systems", IEEE CAS Workshop on Wireless Communications and Networking, Pasadena, September 2002.

[13] Merrill W., Newberg F., Girod L., and Sohrabi K. "Battlefield Ad-Hoc LANs: A Distributed Processing Perspective", $29^{\text {th }}$ Annual GOMATech Conference, Monterey, March 2004.

[14] Savvides A., Han C., Srivastava M.B., "Dynamic FineGrained Localization in Ad-Hoc Networks of Sensors", MobiCom'01, Rome, Italy, July 2001, pages 104-111.

[15] Thisted, R., Elements of Statistical Computing: Numerical Computation, CRC Press, 1988.

[16] Welch G. and Bishop G. "SCAAT: Incremental Tracking with Incomplete Information".

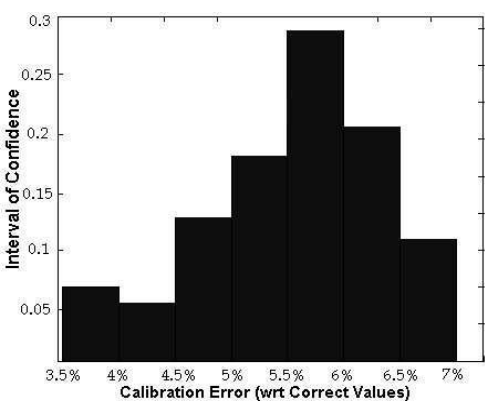

Figure 9. The confidence interval of the calibration error. Proceedings of ACM SIGGRAPH, Los Angeles, Aug 1997, pages 333-344.

[17] Whitehouse K. and Culler D. "Calibration as Parameter Estimation in Sensor Net-works". WSNA'02), Atlanta, 2002, pages 59-67. 